\title{
Nanocrystal seeding: A low temperature route to polycrystalline Si films
}

J. R. Heath, S. M. Gates, and C. A. Chess

IBM T. J. Watson Research Center, Yorkiown Heights, New York 10598

(Received 27 January 1994; accepted for publication 27 April 1994)

A novel method is presented for growth of polycrystalline silicon films on amorphous substrates at temperatures of $540-575^{\circ} \mathrm{C}$. Grain nucleation and grain growth are performed in two steps, using Si nanocrystals as nuclei ("seeds"). The nanocrystal seeds are produced by excimer laser photolysis of disilane in a room temperature flow cell. Film (grain) growth occurs epitaxially on the seeds in a separate thermal chemical vapor deposition (CVD) step, with growth rates 10-100 times higher than similar CVD growth rates on crystal Si. Grain size and CVD growth rates are dependent on seed coverage, for seed coverage $<0.2$ monolayers.

A significant challenge to thin film technology involves growth of crystalline films on amorphous substrates. High temperature annealing or nucleation is normally required, which can limit the choice of substrate. Grain sizes and grain boundaries within the film are poorly controlled. An important example is polycrystalline $\mathrm{Si}$ (poly-Si) grown on $\mathrm{SiO}_{2}{ }^{1-3}$ or on Corning 7059 glass, 4 with applications in thin film transistor ${ }^{6,7}$ and other technologies. Here, we use nanocrystalline particles (NC) to nucleate the growth of poly-Si on amorphous substrates, and call the method nanocrystal seeding for chemical vapor deposition (NC/CVD). Our objectives are (1) reduced growth temperature $(T)$; (2) template relationship between the NC seed lattice and the polycrystalline film; and (3) process control over film grain size.

The NC/CVD method is illustrated in the inset to Fig. 1. In the seeding step (I), the amorphous substrate is coated with NC seeds to a desired coverage ( $(\Theta)$. In the CVD step (II), the seeds are nucleation sites for thermal CVD of a polycrystalline film. Homoepitaxy on Si nanocrystals occurs, and the initial $\mathrm{NC} \theta$ leads directly to grain size control in the film. The growth rate (GR) for NC/CVD depends on $\Theta$, and can be 10-100 times higher than the corresponding GR in thermal Si homoepitaxy at the same $T$.

Figure 1 shows the instrument used for NC/CVD. The Si NC synthesis utilizes a gas flow cell at room temperature, as described elsewhere. ${ }^{8}$ A $5 \% \mathrm{Si}_{2} \mathrm{H}_{6}$ in He precursor gas plus $5 \% \mathrm{H} / \mathrm{Ar}$ mixture (10-20 Torr total pressure) is photolyzed by an unfocused ArF excimer laser beam $(>300 \mathrm{~mJ} /$ pulse, $6.4 \mathrm{eV}, 40 \mathrm{~Hz}$ ) directed down the cell axis. The photolysis region is stirred to promote the homogeneity of each process (photolysis, nucleation, particle growth, and laser annealing) leading to crystalline Si particles. The total flow rates and disilane partial pressure are $100-150 \mathrm{secm}$ and 0.03 Torr, respectively. Step II consists of chamber evacuation, and heating the substrate to the growth $T$. After annealing the $\mathrm{NC}$ seeds for $5-10 \mathrm{~min}$; the $\mathrm{Si}_{2} \mathrm{H}_{6}$ pressure is set for the desired growth time. The substrates used here are Si wafers containing $600 \AA$ of $\mathrm{SiO}_{2}$.

Two transmission electron microscope (TEM) crosssection micrographs of thin poly-Si films grown by NC/CVD appear in Fig. 2. Growth conditions were identical (see figure caption) except for $\mathrm{NC}$ seed coverages, which were 0.01 monolayers (ML) for the top (thin) film, and 0.1 ML for the bottom (thicker) films. Grain size and film thickness are comparable for both films, and the top film exhibits surface roughness of $=10 \AA$. All NC/CVD films discussed here exhibited excellent adherence and structural integrity.

Si NC seeds were collected on an amorphous carbon coated grid, and analyzed for size, areal density (coverage), and crystallinity in a Philips EM420 TEM. The NCs typically deposited as islands of $3-10$ particles. Crystallinity was checked, at low coverage, by selected area electron diffraction (SAD) in the TEM. Lattice imaging of (111) planes revealed the size range to be $40-150 \AA$. Coverage was calibraled by seeding grids for various times. Thick nonadherent coatings of NCs were deposited on (100) oriented Si wafers, and crystallinity was verified by $x$-ray powder diffraction (XPD). Raman scattering from NCs on sapphire revealed the characteristic diamond lattice Si longitudinal optical (LO) phonon mode at $521 \mathrm{~cm}^{-1}$. No evidence for amorphous material was observed. Infrared spectra of NCs demonstrated hydrogen termination of the surface.

Crystallinity of thick poly-Si films $(\simeq 1-10 \mu \mathrm{m})$ was characterized by TEM SAD on cross-sectioned films, x-ray

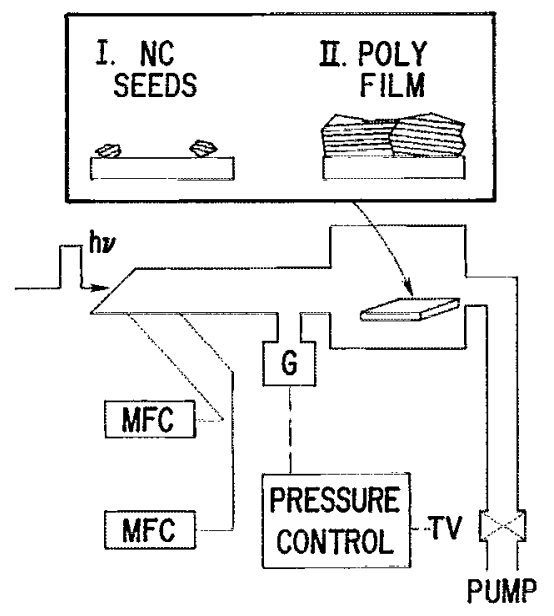

FIG. 1. Instrument for NC/CVD. During seeding, step I, the gas flow is left to right controlled by the MFCs (mass flow controllers). One MFC, controls the $5 \% \mathrm{Si}_{2} \mathrm{H}_{6}$ in $\mathrm{He}$ flow, one controls the inert gas flow. The pressure $(P)$ is 10 Torr, detected by the gauge $(G)$ and set by the $P$ controller using the throttle valve (TV). During CVD, step $\mathrm{II}$, the sample temperature is mertsured by a thermocouple or pyrometer (not shown), and the pressure is set manually, with the TV open. 

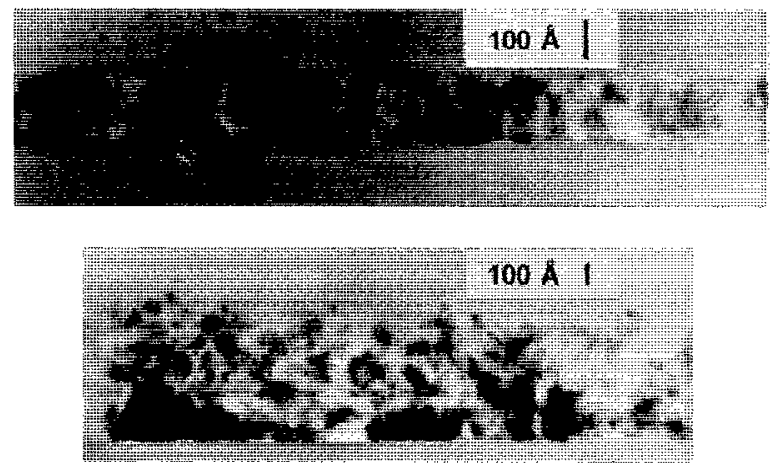

FIG. 2. TEM micrographs of cross-sectioned films grown on $\mathrm{SiO}_{2}$ on $\mathrm{Si}$ wafers. Growth conditions were identical, except for the NC seed coverages, which were $0.01 \mathrm{ML}$ (top) and $0.1 \mathrm{ML}$ (bottom). Growth $T$ was $570^{\circ} \mathrm{C}$, $\mathrm{Si}_{2} \mathrm{H}_{63} P$ was $5 \times 10^{-4}$ Torr, and time was $9 \mathrm{~min}$.

diffraction (XRD), and Raman scattering. The latter exhibited an LO phonon mode with varying amounts of broadening and a $2-10 \mathrm{~cm}^{-1}$ blue shift, probably due to strain (not grain size). An XRD pattern from a film of $15 \mu \mathrm{m}$ thickness grown on $\mathrm{SiO}_{2}$ on $\mathrm{Si}(100)$ appears in Fig. 3. The dashed lines indicate the reflection intensities expected for a statistical distribution $(220: 111=2: 3)$. The anomalously strong (220) reflection $(220: 111=6: 1)$ is indicative of preferred film growth along (110).

Grain sizes were measured by TEM and scanning electron microscopy on cross-sectioned films, with good correlation. Both techniques revealed vertical grain boundaries. A cross section of a $10-\mu$ m-thick NC/CVD film grown on a $\sim 0.5-\mu \mathrm{m}$-thick seed layer revealed a grain size near $100 \AA$ at the base of the film, which gradually increased to around $0.1-0.2 \mu \mathrm{m}$ at the top of the film. For thick films grown near $550^{\circ} \mathrm{C}$, atomic force microscopy (AFM), TEM, and SEM indicated an approximately $0.05 \mu \mathrm{m}$ surface roughness. AFM also revealed features on the film surface which correlated well with grain size measurements from TEM and SEM.

Average NC/CVD growth rates (GR) were estimated by dividing the final film thickness by the CVD growth time. Film thicknesses measured by TEM, SEM, secondary ion mass spectrometry depth profiling, and $\alpha$-step measurements

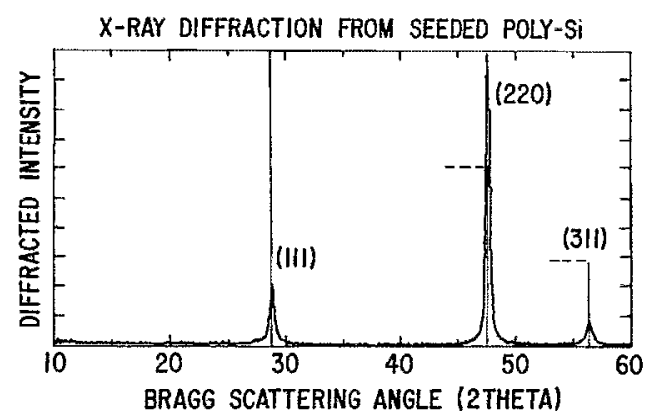

FIG. 3. XRD pattern of a thick (15 $\mu \mathrm{m})$ poly-Si film. The dashed horizontal lines and thin vertical lines coincident with the observed peaks represent statistically expected peak intensities from a randomly oriented crystalline sample. The anomalously strong (220) feature argues for preferred growth along (110). The presence of amorphous $\mathrm{Si}$ would be expected to yield a broad diffraction signal near $15^{\circ}-25^{\circ}=2 \mathrm{~A}$.

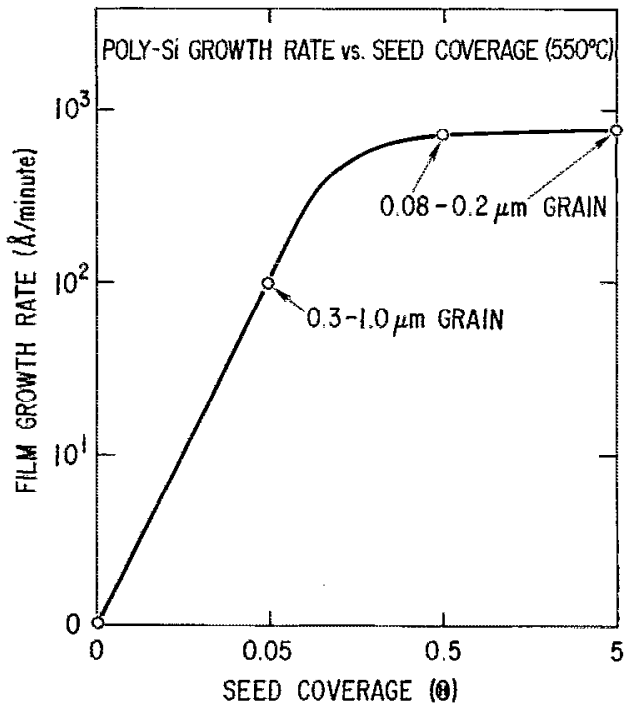

FIG. 4. Plot of $\log ($ film growth rate) vs. $\log ($ seed coverage) for an isothermal film growth series, each grown for $2 \mathrm{~h}$. Grain size and growth rate reach saturation levels at $=0.2$ monolayers seed coverage. For comparison, $\mathrm{Si}_{2} \mathrm{H}_{t}$ thermal CVD on Si(100) proceeds at about $5 \AA /$ min at $550^{\circ} \mathrm{C}$ (Ref. 11 ).

correlated well. Initial NC seed coverage was varied from $\Theta=0$ (the control) to several ML. Figure 4 summarizes the effect of $\Theta$ on average GR and grain size, for films grown at $550^{\circ} \mathrm{C}$ for two hours each. The point at the origin is the control for which all conditions were identical, except that the seeding step was omitted $(\Theta=0)$. In the NC/CVD technique, the physical processes of grain nucleation and growth are separated. The observation that no film grows under the control condition argues that nucleation is the rate limiting step to poly-Si film growth, and that $T \simeq 600^{\circ} \mathrm{C}$ is required to induce nucleation under conventional growth conditions. Below $530^{\circ} \mathrm{C}$, the NCs promote film growth, but the final film is amorphous. We conclude that from $500-575^{\circ} \mathrm{C}$ (the range investigated here), the rate limiting step for poly-Si growth via $\mathrm{NC/CVD}$ is hydrogen desorption from the $\mathrm{NC}$ or poly-Si surface, similar to other thermal low temperature epitaxial (LTE) processes on $\mathrm{Si}^{9}{ }^{9-12}$

The dependence of growth rate on $\Theta$ is not surprising, but the magnitude of that dependence, and the very high GR obtained are surprising. Using $\mathrm{Si}_{2} \mathrm{H}_{6}$, the GR observed for LTE at $550^{\circ} \mathrm{C}$ on single crystal $\mathrm{Si}(100)$ is $\simeq 5 \AA / \mathrm{min},{ }^{11} \mathrm{com}$ pared to $\simeq 800 \AA / \mathrm{min}$ (Fig. 4). Lateral step propagation is thought to be the dominant mechanism is Si epitaxy from $\mathrm{Si}_{2} \mathrm{H}_{6}$ at higher $T_{.}^{12}$ The NC seed coating is characterized by an extremely high surface area, and those surfaces exhibit a roughness with a length scale of tens of $\AA$ s. Thus, the stepdensity is very high, and we believe this roughness efficiently promotes film growth. This mechanism is currently under investigation.

${ }^{1}$ C. A. Dimitriadis, P. A. Coxon, and N. A. Economou, Appl. Phys. Lett. 63, 943 (1993).

${ }^{2}$ D. H. Choi, K. Shimizu, O. Sugiura, and M. Matsumura, Jpn. J. Appl. Phys. 31, 4545 (1993). 
${ }^{4}$ H. Kuriyama, T. Kuwahara, S. Ishida, T. Nohda, K. Sano, H. Iwata, S. Noguehi, S. Kiyama, S. Tsuda, S. Nakano, M. Osumi, and Y. Kuwano, Jpn. J. Appl. Phys. 31, 4550 (1993).

${ }^{5} \mathrm{G}$. Liu and $\mathrm{S}$. J. Fonash, Appl. Phys. Lett. 62, 2554 (1993).

${ }^{6}$ G. Liu and S. J. Fonash, Jpn. J. Appl. Phys. 30, L269 (1991).

${ }^{7}$ I. Yuudasak and $H$. Ohshima, Mater. Res. Soc. Symp. Proc. 182, 333 (1990).

A. Chiang, SPIE 1815, 128 (1992).
${ }^{9} \mathrm{~J}$. Jasinski and F. K. LeGoues, Chem. Mater. 3, 989 (1991).

${ }^{10}$ S. M. Gates and S. K. Kulkmi, Appl. Phys. Lett. 58, 2963 (1991).

${ }^{11}$ B. S. Meyerson, F. J. Himpsel, and K. J. Uram, Appl. Phys. Lett. 57, 1034 (1990).

${ }^{12}$ S. M. Mokler, W. K. I.in, N. Ohtani, and B. A. Joyce, Appl. Phys. Lett. 60, 2255 (1992)

${ }^{13}$ W. K. Liu, S. M. Mokler, N. Ohtani, J. Zhang, and B. A. Joyce, J. Appl. Phys. 71, 5168 (1992). 